\title{
DIFERENÇAS NA CONSTITUIÇÃO DO PLASMA RICO EM PLAQUETAS: INFLUÊNCIA NOS RESULTADOS TERAPÊUTICOS
}

\author{
DIFFERENCES IN THE CONSTITUTION OF PLATELET RICH PLASMA: \\ INFLUENCE ON THERAPEUTIC RESULTS
}

\author{
Géssica Cantadori Funes Arenas ${ }^{1}$ \\ Rossana Catanhede Farias de Vasconcelos ${ }^{2}$ \\ Clayton Rodrigues da Silva ${ }^{3}$
}

RESUMO: O plasma rico em plaquetas inicialmente se trata de uma técnica laboratorial, utilizada para fins de diagnóstico e hemoterapia, posteriormente começou a ser empregada para fins terapêuticos, como um bioestimulador na medicina regenerativa. As diferentes técnicas e nomenclaturas utilizadas para a obtenção do plasma rico em plaquetas para fins terapêuticos, dificultam a conclusão de evidências científicas quanto aos benefícios dessa terapia biológica nas diversas áreas de saúde. Foram avaliadas diferentes técnicas de plasma rico em plaquetas, de sistema aberto e fechado, classificações atuais e publicações com diferentes resultados para o mesmo tratamento proposto. A concentração de plaquetas, presença e concentração de leucócitos, tipo de ativação interferem diretamente nos resultados obtidos nos tratamentos. Este artigo sugere a percepção das diferentes técnicas e suas particularidades, compreende que o termo PRP - plasma rico em plaquetas não deve ser genérico. As classificações ajudam a guiar a escolha de técnicas que possuem maior probabilidade de resultados positivos. Deixar descrito nos artigos, todos os dados obtidos com o preparo, é importante para avaliação mais ampla do método e da eficácia do PRP.

\footnotetext{
I Géssica Cantadori Funes Arenas. Bacharel em Ciências Biológicas - Universidade São Judas Tadeu - USJT; Pós Graduação latu senso em Análises Clínicas e Toxicológicas - Universidade Guarulhos UNG; Pós Graduação latu senso em Estética Facial e Corporal com título de Dermaticista ${ }^{\circledR}$ Instituto IBECO, Faculdade Mário Schernerg; Pós Graduação latu senso em Fitoterapia - AVW faculdade Integrada. Pós Graduanda latu senso em Anti-Aging (Unyleya). Email: gcfabiol@yahoo.com.br.

2 Rossana Catanhede Farias de Vasconcelos. Médica Dermatologista. Dermatoloy Depertment Universidade de Santo Amaro (UNISA)- São Paulo-SP.

${ }^{3}$ Clayton Rodrigues da Silva. Biomédico - Universidade de Mogi das Cruzes; Pós Graduação em Citopatologia Ginecológica - Universidade dos Bandeirantes - UNIBAN; MBA em Gestão em Saúde Hospitalar - Universidade Nove de Julho - UNINOVE; Pós Graduação em Administração Hospitalar - Instituto de Pesquisa e Educação em Saúde de São Paulo - IPESSP; Pós Graduação em Auditoria de Serviços de Saúde - Instituto de Pesquisa e Educação em Saúde de São Paulo - IPESSP; Pós Graduação em Informática em Saúde - Universidade Federal de São Paulo - UNIFESP; Pós Graduação em Perícia Criminal e Ciências Forenses - Instituto de Pós Graduação e Graduação IPOG.
} 
Palavras-chave: Plasma Rico em Plaquetas; Regeneração; Fatores de Crescimento; Terapia Regenerativa.

ABSTRACT: Platelet-rich plasma is initially a laboratory technique, used for diagnostic and hemotherapy purposes, later it started to be used for therapeutic purposes, as a biostimulator in regenerative medicine. The different techniques and nomenclatures used to obtain platelet-rich plasma for therapeutic purposes, make it difficult to conclude scientific evidence regarding the benefits of this biological therapy in different health areas. Different techniques of platelet-rich plasma, open and closed system, current classifications and publications with different results for the same proposed treatment were evaluated. Platelet concentration, presence and concentration of leukocytes, type of activation directly interfere with the results obtained in the treatments. This article suggests the perception of different techniques and their particularities, and understands that the term PRP - plateletrich plasma should not be generic. Classifications help guide the choice of techniques that are most likely to have positive results. Leaving all the data obtained with the preparation described in the articles is important for a broader evaluation of the method and the effectiveness of the PRP.

Keywords: Platelets Rich Plasma. Regeneration. Growth Factors. Regenerative Therapy.

\section{INTRODUÇÃO}

O plasma rico em plaquetas (PRP) para fins terapêuticos não transfusionais, é um produto derivado do processamento laboratorial do sangue autólogo, coletado e processado momentos antes da aplicação. A obtenção desse concentrado de plaquetas, rico em fatores de crescimento que estão localizados no interior dos grânulos alfa, tem a finalidade de estimular a modulação biológica e regeneração tecidual (PONTUAL \& MAGINI, 2004), (LIEBERMAN et al., 2002), (SIERRA et al., 20II), (LORENZI T.F, 2003).

O método PRP consiste em uma técnica laboratorial praticada em bancos de sangue (HOSPITAL SÍRIO LIBANÊS, 2019) e análises clínicas, realizada por profissional habilitado e especializado. $O$ teste de agregação plaquetária (HOSPITAL ALBERT EINSTEIN, 2018) um dos exames da hematologia e hemostasia, realiza a técnica do PRP para fins de diagnóstico.

O conhecimento das diferentes técnicas de centrifugação, que inclui força centrífuga relativa e velocidade de rotação, para separar as partículas de uma solução (LABORATÓRIO MULTIUSUÁRIO DE ESTUDOS EM BIOLOGIA I, 2012), está presente nas rotinas laboratoriais, e se faz importante para a exatidão dos resultados para fins de diagnósticos e pesquisa. A partir deste conhecimento e experiência em laboratórios clínicos e hemoterápicos que se desenvolveu as técnicas de PRP para fins terapêuticos (PONTUAL \& MAGINI, 2004).

As diferentes técnicas e nomenclaturas utilizadas para a obtenção do PRP para fins terapêuticos, dificultam a conclusão de evidências científicas quanto aos benefícios dessa terapia biológica nas diversas áreas de saúde. 


\section{METODOLOGIA}

É notável que ocorre divergência de resultados em toda a literatura disponível, leva-se em consideração como o plasma rico em plaquetas é processado e de acordo com a técnica utilizada os resultados mostram benefícios importantes ou insuficiência.

Este estudo comparou e avaliou os dados de diferentes técnicas encontradas na literatura mundial, para a obtenção de plasma rico me plaquetas sendo sistemas abertos e fechados. Observou as divergências quanto a nomeação do procedimento, as informações contidas nas publicações quanto ao detalhamento e resultados obtidos através das técnicas e como isso pode influenciar na conclusão da eficácia ou não do plasma rico em plaquetas nas diversas aplicações encontradas na literatura.

\section{I. Origem e Diferenças nas Técnicas de Plasma Rico em Plaquetas}

Após os protocolos estudados até o ano 2000, Anitua (2003) apresentou outra técnica (PONTUAL \& MAGINI, 2004), um sistema com centrífuga digital de laboratório clínico, a I8oorpm por 8 minutos, onde o plasma obtido era separado em quatro fases, sendo a mais próxima do concentrado de hemácias, a porção mais rica em plaquetas e leucócitos.

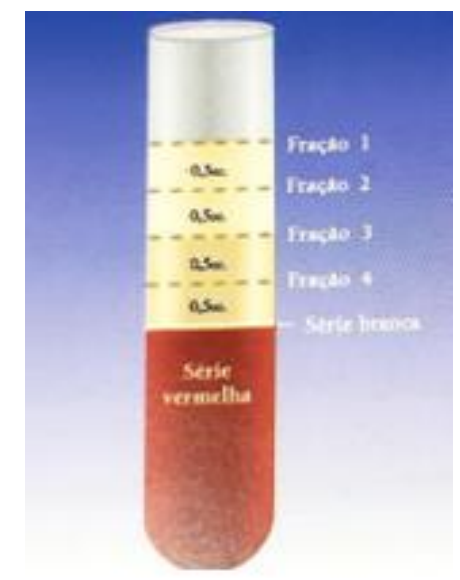

Figura I - Separação das frações de plasma, segundo Dr Anitua. (Imagem do livro Plasma Rico em Plaquetas PRP e Fatores de Crescimento, 2004).

\subsection{Protocolo Cantadori (2009) - Técnica manual ou Sistema Aberto}

Protocolo Cantadori (2009), é uma técnica registrada: 508.102 na Biblioteca Nacional do RJ, criada por Géssica Cantadori Funes Arenas, bióloga, para realização da técnica PRP - plasma rico em plaquetas para fins terapêuticos não transfusionais (VASCONCELOS et al., 2015). A técnica é realizada conforme as normas de biossegurança (AGÊNCIA NACIONAL DE VIGILÂNCIA SANITÁRIA, 2006), e demais cuidados antissepticos (CAN, 2015), (JSM, 2015), possui as concentrações de plaquetas, leucócitos e tipo de ativação adequada conforme as atuais classificações encontradas na literatura, como a PAW Classification (DELONG et al., 2012). 


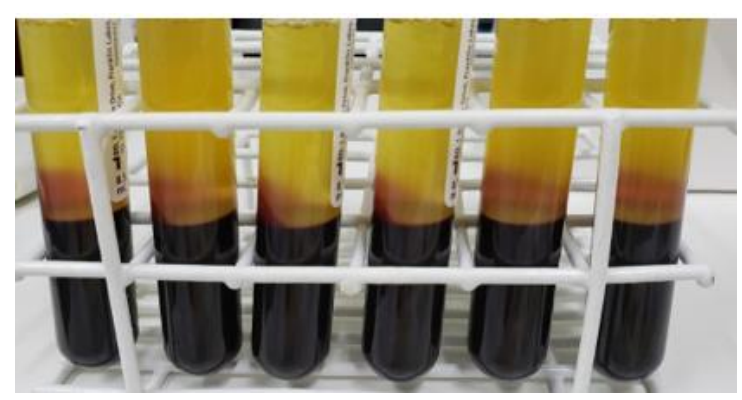

Figura 2 - Protocolo Cantadori (2009) - $60 \mathrm{ml}$ de sangue venoso colhido em tubo estéril à vácuo com $\mathrm{ACD}-\mathrm{A}$. Preparo do PRP. As Plaquetas e Leucócitos se encontram em maior concentração próximo à linha das hemácias, devido peso molecular e densidade. O volume a ser aplicado será de $3 \mathrm{ml}$ de PRP.

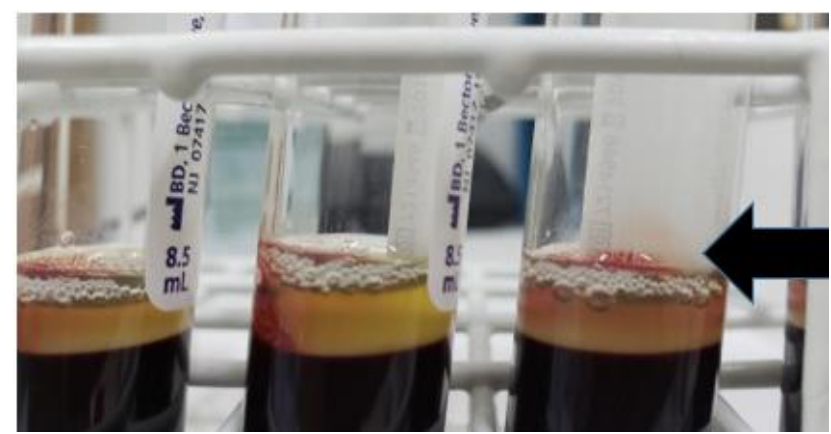

Figura 3 - Protocolo Cantadori (2009). O PPP -Plasma Pobre em Plaquetas é separado. As Plaquetas somente são coletadas nesta porção, quanto menos PPP (amarelo), maior será a concentração obtida. A "nuvem” de leucócitos também é encontrada nesta mesma área e deve ser coletada. É o chamado buffy coat.

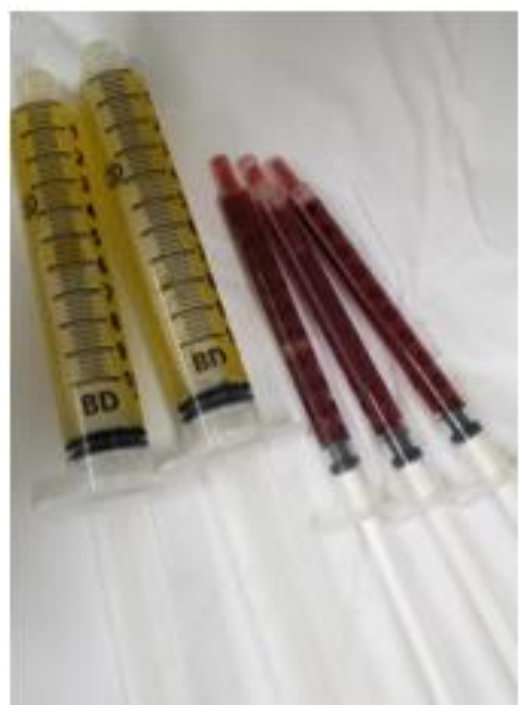


Figura 4 - Protocolo Cantadori (2009). PPP- Plasma Amarelo - Plasma em maior volume, baixo índice de plaquetas e células. PRP - Plasma avermelhado - Devido a mínima presença de hemácias jovens, Nesta faixa é onde se concentram as plaquetas e leucócitos após a centrifugação. O volume de PRP é cerca de $5 \%$ do volume total colhido.

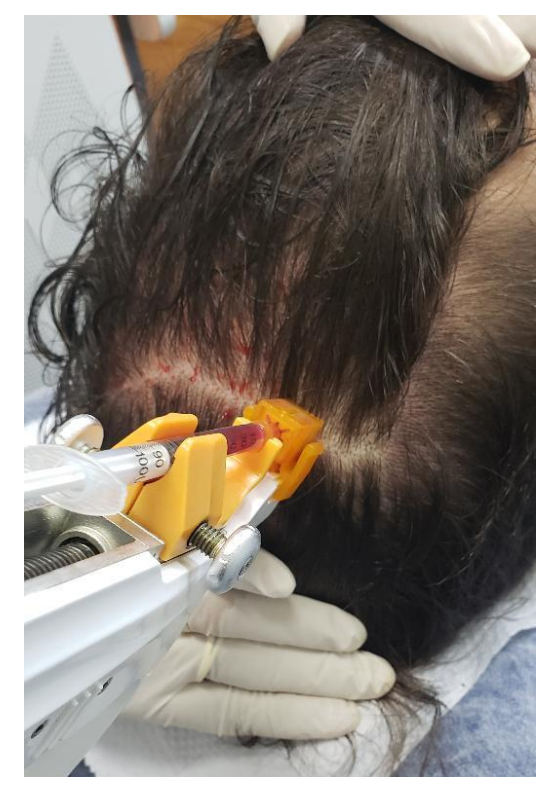

Figura 5 - Aplicação do PRP - Protocolo Cantadori, em tratamento de alopecia androgenética, atraves de equipamento de injeção pressurizada.
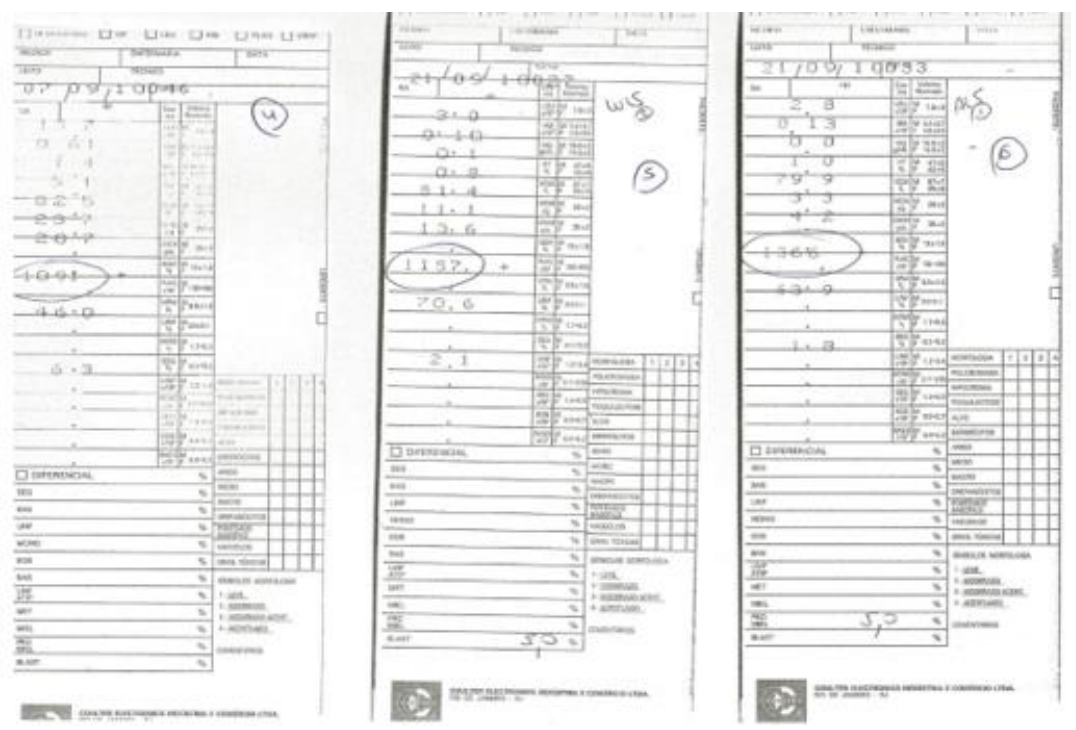

Figura 6 - Protocolo Cantadori (2009). Dados de concentrados, PRP. Valor xio ${ }^{3}$. I. Contagem de Plaquetas: as 3 amostras acima apresentam contagem maior que I.00o.0oo plaquetas/ul e não maiores que r.80o.00o/ul. 2. Leucócitos: presentes, porém em baixas concentrações, igual ou menor à basal. Equipamento: Contador Hematológico Couter T89o-Beckman couter. 


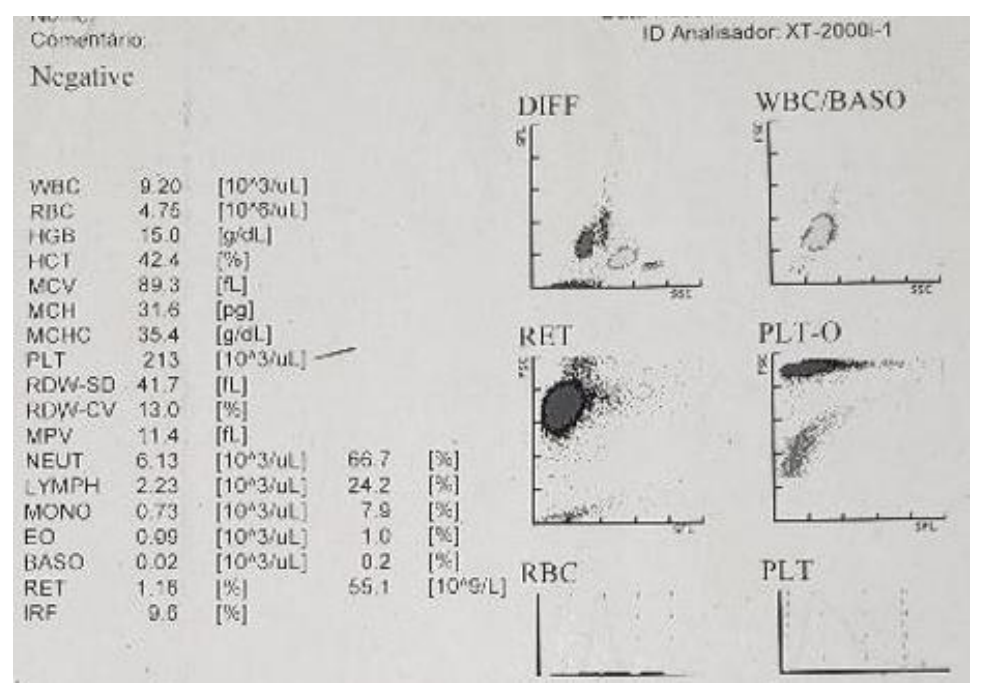

Figura 7 - Protocolo Cantadori (2009). A. Contagem de amostra basal. Plaquetas: 213 xio3/ul, Leucócitos: 9,20 xio3/ul. Equipamento: Roche XT 20ooi-I.

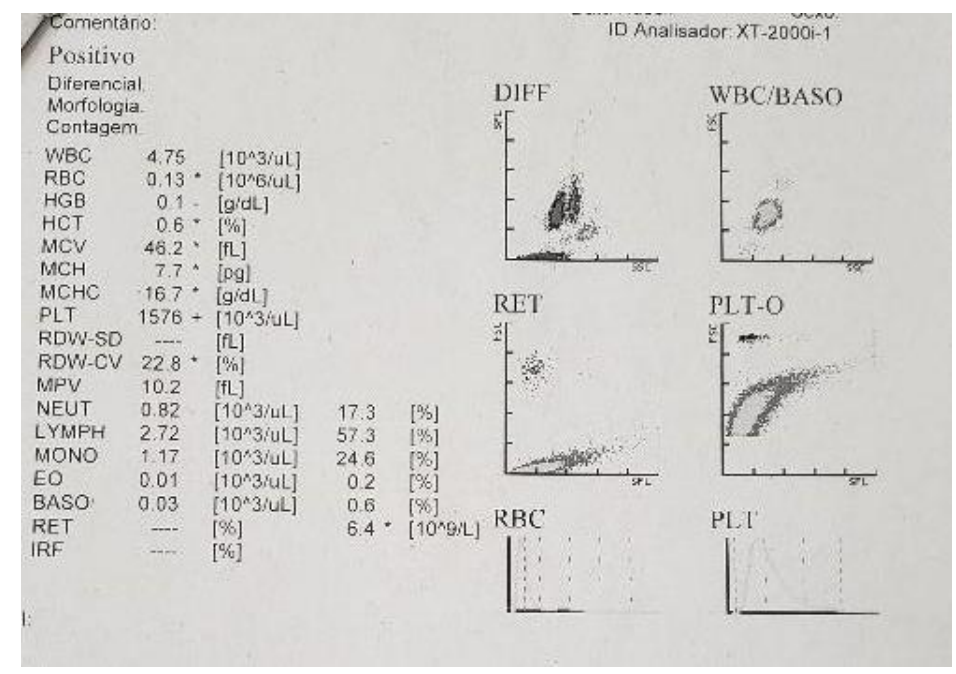

Figura 8 - Protocolo Cantadori (2009). B. Contagem do PRP. Plaquetas: $1576 \times 10^{3} / \mathrm{ul}$, Leucócitos: 4,75 $\mathrm{xIO}^{3} /$ ul. Equipamento: Roche XT 200oi-I. 


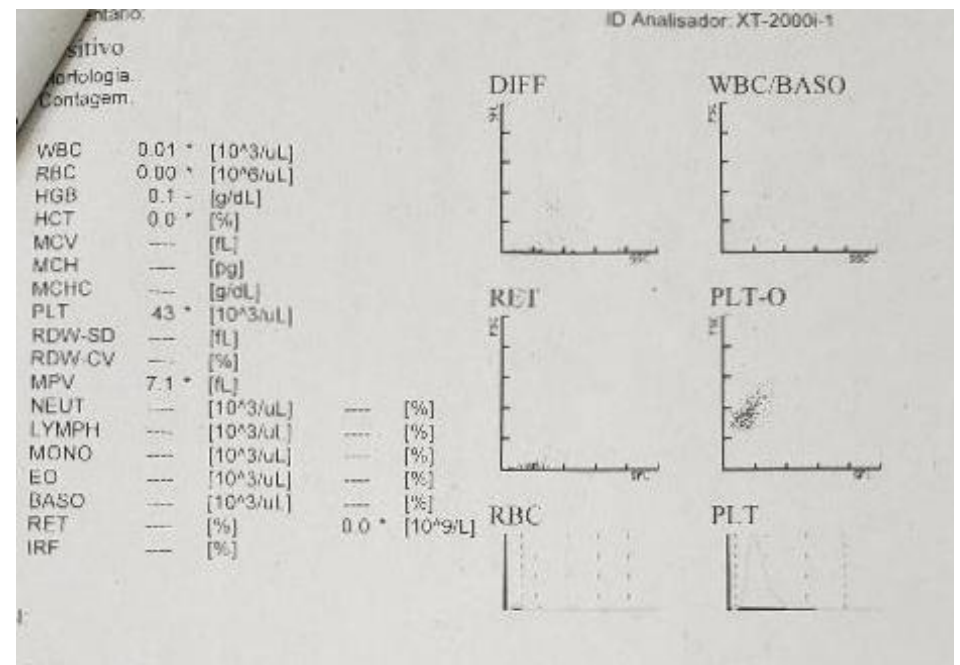

Figura 9 - Protocolo Cantadori (2009). Contagem do PPP. A maior concentração de plaquetas está no PRP. Equipamento: Roche XT 20ooi-I.

\subsubsection{Sistemas Fechados (Kits comerciais)}

Comercialmente foram lançados, os sistemas chamados "sistemas fechados", onde o sangue é processado em dispositivos sem o contato com o meio ambiente (FITZPATRICK et al., 2017). Esses sistemas apresentam diferenças importantes nas concentrações de plaquetas, leucócitos e presença significativa ou não de hemácias no produto final, chamado de PRP.

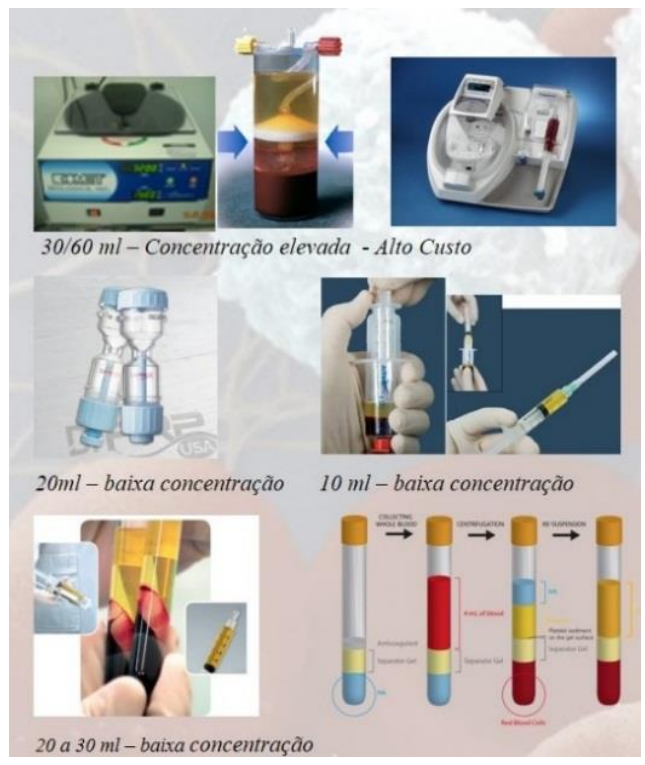

Figura io - Modelos de Sistemas Fechados - Kits Comerciais. (Fontes: Zimmerbiomet/Medtronic Magelan/ Dr PRP USA/ Arthrex/ Regenlab).

Todos os produtos são chamados de PRP apesar de observarmos na literatura grande diferença entre eles, alguns realmente podem ser considerados ricos em 
plaquetas, outros porém, apresentam-se diluídos por grande porção de plasma sanguíneo. Alguns utilizam uma pequena quantidade de sangue para o processo, o que implica na concentração de plaquetas, estando abaixo de r.ooo.ooo plaquetas/ul (DELONG et al., 2012), (D'ELIA et al., 2009), (FOSTER et al., 2009), (MARX 2001, 2004), (EPPLEY et al., 2004).

\subsection{Aspéctos técnicos e classificações}

Foster et al (20II), define na sua pesquisa que o PRP possui concentração por volta de 1.000 .000 plaquetas/ul em $5 \mathrm{ml}$ de plasma, associa a esta concentração a melhora na cicatrização e relata que as variáveis no preparo do $\mathrm{PRP}$, afetam a atividade biológica nos tecidos.

Uma das mais consideradas classificações atualmente é a PAW Classification (2012). Os autores avaliaram na literatura, as diferentes concentrações de plaquetas obtidas no PRP, tipos de ativação, presença de anestésicos no local da aplicação, se o achado de leucócitos ou não seria um interferente e como poderia alterar os resultados. Metais são prejudiciais se utilizados com o PRP, pois comprometem a adesão plaquetária, os tubos mais apropriados são os de polipropileno, os de vidro não revestidos podem ativar as plaquetas precocemente, alterando os resultados. Concluíram que essa metodologia e classificação oferece uma maneira eficaz para obtenção de melhores resultados e novas pesquisas.

Pereira et al (2012), estudou sete protocolos de obtenção de PRP, concluindo que o mais eficaz possui baixa concentração de leucócitos, alta de plaquetas e mínimo de hemácias.

DEPA Classification (2016), os autores tomaram como base classificações utilizadas em terapia celular e avaliaram a dose de plaquetas injetadas, eficiência de produção, pureza do PRP e ativação. Identificaram que nenhum dos dispositivos estudados foi capaz de recuperar mais de $90 \%$ das plaquetas, em relação à pureza, a maioria possui contaminação por hemácias, apenas 3 mostraram ausência total de hemácias, Curasan, Regen e método Kushida et al., porém apresentaram concentração baixa de plaquetas e leucócitos. Isso mostra que o termo PRP puro somente está relacionado com a ausência de outras células, e não significa que o PRP é eficaz, já que a literatura mostra um índice de eficiência nas técnicas de PRP que atingem mais Iooo.ooo plaquetas/ul ((DELONG et al., 2012), (D'ELIA et al., 2009), (FOSTER et al., 2009), (MARX 2001, 2004), (EPPLEY et al., 2004).

\section{RESULTADOS E DISCUSSÃO}

Em grande parte da literatura é citado o nome "PRP - plasma rico em plaquetas” sem especificações do método utilizado para a realização da técnica, subentende-se que PRP é como um produto único, onde se obtém com a técnica sempre os mesmos resultados, o que não ocorre. PRP é uma técnica que pode ser realizada de várias maneiras, e dependendo da metodologia aplicada, os resultados são variados, promovendo eficácia ou não. Alguns sistemas e técnicas são encontrados com frequência nos estudos referentes à eficácia do PRP, como, o Sistema GPS ${ }^{\circledR}$ III - Zimmer Biomet; Regen $\mathrm{BCT}^{\circledR} / \mathrm{ACR}^{\circledR}$ - Regenlab, $\mathrm{ACP}^{\circledR}$ - 
Arthex, técnicas por aférese, ROOYA GEN ${ }^{\circledR}$ - Kashani Laboratory, Dr PRP-Kit ${ }^{\circledR}$ REMI Laboratory Instruments e técnicas manuais, como a Protocolo Cantadori (2009).

Tahririan et al (2016), considerando a importância das alterações degenerativas e processo inflamatório no manguito rotador, realizaram o estudo do PRP - na eficácia do tratamento nas tendinopatias crônicas. O Sistema utilizado, de obtenção de PRP, resultou na concentração de plaquetas entre $1325 \pm 121.2 \times \mathrm{IO}^{3} / \mu \mathrm{L}$. Concluíram neste estudo que a injeção de PRP reduz a dor e melhora da amplitude em pacientes com ruptura parcial do tendão, já um estudo de Schwitzguebel et al (2019), randomizado, duplo-cego, nível I de evidência, comparou os efeitos das infiltrações de PRP e solução salina (placebo) na melhora da cicatrização, para o tratamento do supraespinhal intersticial. Os autores não observaram diferença entre os grupos, mas no estudo não foram informadas as concentrações de plaquetas e leucócitos obtidas pelo método escolhido, nem informações sobre a ativação realizada do PRP antes da aplicação.

Ayatollahi et al (2017), realizaram um estudo em alopecia androgenética, utilizando um sistema para obtenção de plaquetas Os dados de contagem mostraram a concentração de plaquetas de $1,6 \mathrm{x}$ o basal, os autores relacionam a esta baixa concentração os resultados negativos que obtiveram no estudo. Em comparação, Vasconcelos et al (2015), realizou um estudo em alopecia androgenética masculina e feminina, utilizando PRP, cuja técnica utilizada foi a Protocolo Cantadori 2009 (registro 508.102 na Biblioteca Nacional do RJ), onde as concentrações de plaquetas se apresentam acima de r.ooo.ooo plaquetas/ul e baixa concentração de leucócitos, ativação com gluconato de cálcio ro\% e trombina autóloga. Concluíram ao final da pesquisa que o PRP utilizado nesta pesquisa, melhora a resposta terapêutica e que se mostrou ser uma alternativa interessante e eficaz para o tratamento da alopecia androgenetica (VASCONCELOS et al., 2015). O mesmo autor, Vasconcelos et al (2016), utilizou esse mesmo protocolo e técnica para outro estudo, avaliando os resultados no tratamento de difícil controle da alopecia areata, onde conclui também resultados favoráveis utilizando o PRP no tratamento.

Schulz et al (2018), estudou o lisado de plaquetas para o tratamento em tendões e comparou com alguns dispositivos que chamou de soro humano, concentrado por aférese e após descongelamento. Os produtos apresentaram índices de plaquetas diferentes, sendo que o enriquecimento maior foi $3,8 \mathrm{x}$ o sangue basal no sistema aférese. Um dos sistemas apresentou concentração $1,9 \mathrm{x}$ e outro $0,7 \mathrm{x}$ menor em comparação ao sangue basal. Os leucócitos foram reduzidos em todos os sistemas e a quantificação dos fatores de crescimento, apresentou baixas concentrações em todos os sistemas comerciais em comparação com aférese.

Stevens \& Khetarpal (2018), estudou 9 métodos diferentes de obtenção de PRP para o tratamento de alopecia androgenética. Verificou que existem grandes diferenças entre as metodologias e resultados. Avaliou a concentração de plaquetas, tipo de ativação, protocolo utilizado e resultado. Um dos sistemas apresentou a maior concentração de plaquetas (6-7x o basal) e resultados positivos para o tratamento em seis meses. Quatro deles, não informaram as concentrações de plaquetas, o método que apresentou a menor concentração de plaquetas, 2-3x, relatou resultado negativo ao tratamento. Os autores concluíram que o PRP pode ser uma alternativa eficiente 
de tratamento para a alopecia androgenética, já que de 9 métodos estudados, 7 mostraram resultados positivo.

Rodrigues et al (2018), em um estudo randomizado, avaliou 26 pacientes com alopecia androgenética, onde um grupo $(n=15)$ recebeu 04 injeções, com intervalo de I5 dias, de PRP, obtido por técnica aberta, com média de concentração de Io82xio ${ }^{6}$ plaquetas/ul, leucócitos e metodologia de ativação não foram mencionados. Foram dosados alguns fatores de crescimento, como EGF, PDGF e VEGF. Em conclusão o autor relata que o PRP se mostrou um alternativa favorável no tratamento da alopecia androgenética, mas que a falta de associações entre, contagem de plaquetas, nível de fatores de crescimento e dados clínicos, melhorariam os apontamentos de fatores envolvidos nesta resposta.

Ehrenfest et al (2018), realizou um estudo avaliando as centrífugas usadas nos processos, considerando as vibrações de quatro modelos de centrífugas de bancada na produção de L-PRF (plasma rico em fibrina contendo leucócitos). Concluiu que o nível de vibração da centrífuga, quando excessiva, pode ocasionar danos e até perda de células, o que também interfere na formação da fibrina e coágulo, além da liberação de fatores de crescimento.

Em uma revisão sistemática, Pachito et al (2019), de 15 artigos consultados sobre a ação do plasma rico em plaquetas na ortopedia, apenas 2 mostraram a contagem de plaquetas, aproximadamente, I relatou que as amostram continham um valor próximo a Iooo.ooo de plaquetas/ $\mathrm{ml}$ e presença de leucócitos.

Gentili et al (2020), relacionou resultados em seu estudo abrangendo diferentes técnicas, de sistema fechado e técnica aberta, de PRP, levando em consideração a quantidade de leucócitos, densidade de fibrina, ativação e não ativação, volume de sangue coletado, reagentes utilizados, tempo de rotação e gravidade, temperatura e especificações da centrífuga. $O$ autor avaliou os resultados do $P R P$ ativado e não ativado em diferentes patologias e percebeu que existe diferença de ação conforme o tecido a ser tratado.

Gupta et al (2020), avalia em seu estudo que nem sempre os sistemas fechados garantem concentrações de plaquetas e leucócitos já vistas como eficazes. Neste estudo foi comparado uma técnica manual, ou aberta, com um sistema fechado, onde foram realizadas as contagens de plaquetas e leucócitos após o preparo do PRP. A diferença entre os dois métodos foi significativa, sendo a técnica manual bem superior ao sistema fechado (plaquetas: $12,512 \times 10^{5}$ e $7,254 \times 10^{5}$ respectivamente; e leucócitos: $1,23 \times 10^{3}$ e $7,27 \times 10^{3}$ respectivamente).

Acebes-Huerta (2020) em seu estudo levanta a relevância das classificações citadas como Dohan Ehrenfest e DEPA, no preparo e eficácia do plasma rico em plaquetas, enfatizam que a falta de informações nos trabalhos científicos dificultam não apenas a obtenção de dados sobre a eficácia mas também sobre um aspecto de segurança.

\section{CONCLUSÃO}

Este artigo sugere a percepção das diferentes técnicas e suas particularidades, compreende que o termo PRP não deve ser genérico e sim específico, conforme produto final obtido (YIN et al., 2016), (GILBERTIE et al., 20I8). As classificações 
ajudam a guiar a escolha de técnicas que possuem maior probabilidade de resultados positivos.

Para maior segurança, o procedimento deve ser realizado por profissional especializado, com experiência em processamentos laboratoriais, além das práticas de biossegurança. Ficou claro, portanto, que quando a técnica de PRP utilizada, reproduz as classificações estudadas (concentração de plaquetas em torno de I.000.00o - 1.800.00o plaquetas/ul, baixa concentração de leucócitos e ativação do concentrado (DELONG et al., 2012/ MAGALON et al., 2016)), os autores observam a eficácia terapêutica do PRP.

É importante que sejam relatados nas publicações todos os dados obtidos, incluindo volume de sangue colhido, as concentrações obtidas de plaquetas, leucócitos e hemácias, aditivos, tipo de ativação, quantificações de substâncias, tanto de fatores de crescimento como de produtos do processo inflamatório, volume de PRP injetado e equipamentos utilizados. Esses dados são primordiais para a avaliação da qualidade do PRP e sua eficácia.

\section{REFERÊNCIAS}

ACEBES-HUERTAA A, ARIAS-FERNANDEZA T, BERNARDOA A, MUNOZ-TURRILLASA MC, FERNANDEZ-FUERTESA J, SEGHATCHIANE J, GUTIERREZ L. Platelet-derived bio-products: Classification update, applications, concerns and new perspectives. Transfusion and Apheresis Science. 59,102716. 2020.

AYATOLLAHI A, HOSSEINI $H$, SHAHDI $M$, NASROLLAHI AS, KASHANI MN, YADANGI S, FIROOZ FH, FIROOZ A. Platelet-rich Plasma by Single Spin Process in Male Pattern Androgenetic Alopecia: Is it an Effective Treatment? Indian Dermatol Online J. 8(6):460-464. 2017.

Banco de sangue - Doação Automatizada de Plaquetas. Hospital Sírio libanês. 2019. Disponível em 〈https://www.hospitalsiriolibanes.org.br/hospital/Paginas/doacaoautomatizada-plaquetas.aspx $>$ Acesso em Set. 2020.

C.A, N. VANTAGENS NA UTILIZAÇÃO DE PERFUROCORTANTE COM DISPOSITIVO DE SEGURANÇA PARA A PREVENÇÃO DE ACIDENTES COM MATERIAL BIOLÓGICO NO PRONTO ATENDIMENTO. Biológicas \& Saúde, v. 5, n. I8, 24 nov. 2015.

D’ELIA CO, REZENDE UM, BITAR AC, TATSUI N, PÉCORA JR, CAMANHO GJ. O uso do plasma rico em plaquetas associado ao aspirado de medular óssea na osteotomia tibial tipo Puddu. Rev Bras Ortop.44(6):508-12.2009.

DELONG JM, RUSSELL RP, MAZZOCCA AD. Platelet-Rich Plasma: The PAW Classification System. Arthroscopy. J Arthroscopic Related Surgery. 28(7):998-1009. 2012. 
EHRENFEST DMD, PINTO NR, PEREDA A, JIMÉNEZ P, CORSO MD, KANG BS, NALLY M, LANATA N, WANG HL, QUIRYNEN M. The impact of the centrifuge characteristics and centrifugation protocols on the cells, growth factors, and fibrin architecture of a leukocyte- and platelet-rich fibrin (L-PRF) clot and membrane. Platelets. 29(2): 17I-184. 2018.

EPPLEY BL, WOODELL JE, HIGGINS J. Platelet quantification and growth factor analysis from platelet-rich plasma: implications for wound healing. Plast Reconstr Surg. II4(6):1502-8. 2004 .

FITZPATRICK J, BULSARA MK, MCCRORY PR, RICHARDSON MD, ZHENG MH. Analysis of Platelet-Rich Plasma Extraction Variations in Platelet and Blood Components Between 4 Common Commercial Kits. The Orthopaedic Journal of Sports Medicine. 5(I). 2017.

FOSTER TE, PUSKAS NL, MANDELBAUM BR, GERHARDT MB, RODEO AS. Platelet-Rich Plasma. From Basic Science to Clinical Applications. American Journal of Sports Medicine. v37.II. 2009.

GENTILE P, CALABRESE C, ANGELIS BD, DIONISI L, PIZZICANNELLA J, KOTHARI A, FAZIO DD, GARCOVICH S. Impact of the Diferent Preparation Methods toObtain Autologous Non-Activated Platelet-RichPlasma (A-PRP) and Activated Platelet-Rich Plasma(AA-PRP) in Plastic Surgery: Wound Healing and Hair Regrowth Evaluation. Int. J. Mol. Sci. 2I, 43I. 2020.

GILBERTIE JM, LONG JM, SCHUBERT AG, BERGLUND AK, SCHAER TP, SCHNABEL LV. Pooled Platelet-Rich Plasma Lysate Therapy Increases Synoviocyte Proliferation and Hyaluronic Acid Production While Protecting Chondrocytes From Synoviocyte-Derived Inflammatory Mediators. Front Vet Sci. 5(150):I-9. 2018.

GUPTA V, PARIHAR AS, PATHAK M, SHARMA VK. Comparison of PlateletRich Plasma Prepared Using Two Methods: Manual Double Spin Method versus a Commercially Available Automated Device. Indian Dermatology Online Journal. 2020 .

Hospital Albert Einstein. Manual de Procedimento Técnico - Agregação Plaquetária, 2018.

J. S., M. LAVAGEM DAS MÃOS: ATUAÇÃO DO ENFERMEIRO NO CONTROLE E PREVENÇÃO DE INFECÇÕES NA UNIDADE DE TERAPIA INTENSIVA NEONATAL. Biológicas \& Saúde, v. 5, n. I8, 24 nov. 2015.

KUSHIDA S, KAKUDO N, MORIMOTO N, HARA T, OGAWA T, MITSUI T, KUSUMOTO K. Platelet and growth factor concentrations in activated platelet-rich plasma: a comparison of seven commercial separation systems. J Artif Organs. 2014. 
Laboratório Multiusuário de Estudos em Biologia I. Disponível em: <lameb.ccb.ufsc.br/files/2012/og/A-relação-entre-RPM-e-RCF-ou-Força-G.pdf〉. Acesso em Ago. 2020.

LIEBERMAN J, DALUISKI A, EINHORN TA. The Role of Growth Factors in the Repair of Bone. Biology and Clinical Applications. Investigation performedat the Department of Orthopaedic Surgery, University of California at Los Angeles Medical Center, Los Angeles, California, and the Department of Orthopaedic Surgery, Boston University Medical Center, Boston, Massachuset. J Bone Joint Surg Am.84(6):1032-44. 2002.

LORENZI T.F. Manual de Hematologia Propedêutica e Clínica. $3^{\circ}$ ed. Medsi, SP, 2003.

MAGALON J, CHATEAU AL, BERTRAND B, LOUIS ML, SILVESTRE A, GIRAUDO L, VERAN J, SABATIER F. DEPA Classification: A proposal forstandardising PRP use and a retrospective application of available devices. Open Sport Exerc Med. 2:e0ooo6o. 2016.

Manual de gerenciamento de resíduos de serviços de saúde / Ministério da Saúde, Agência Nacional de Vigilância Sanitária. - Brasília: Ministério da Saúde, 2006. I82 p. - (Série A. Normas e Manuais Técnicos) ISBN 85-334-II76-6. Disponível em : < https://www.anvisa.gov.br/servicosaude/manuais/manual_gerenciamento_residuos. pdf. Acesso em Set. 2020 .

MARX RE. Platelet-rich plasma (PRP): What is PRP and what is not PRP? Implant Dentistry. IO(4):225-8. 2. 2001.

MARX RE. Platelet-rich plasma: evidence to support its use. J Oral Maxillofac Surg. 62(4):489-96.2004.

PACHITO DV, LATORRACA COC, RIERA R. Efficacy of platelet-rich plasma for non-transfusion use: Overview of systematic reviews. Int J Clin Pract.oo:er3402. 2019.

PEREIRA RCF, ZACARIAS GVF, CANTARELLI C. MATOSO M, SILVA GBD, BRASS ET AL. Avaliação de sete protocolos obtenção do plasma rico em plaquetas. Universidade Federal de Santa Maria; 2012.

PONTUAL MAB, MAGINI RS. Plasma Rico em Plaquetas PRP e Fatores de Crescimento das Pesquisas Científicas à Clínica Odontológica. São Paulo: Ed.Santos. p.163-87. 2004 .

RODRIGUES BL, MONTALVÃO SAL, CANCELA RBB, SILVA FAR, URBAN A, HUBER SC,JUNIOR JLRC, LANA JFSD, ANNICHINNO-BIZZACCHI JM. Treatment of male pattern alopecia with platelet-rich plasma: A double-blind 
controlled study with analysis of platelet number and growth factor levels. J Am Acad Dermatol. 80(3):1-9. 2018.

SCHULZ FK, SCHMIDT $T$, UCKERT M, SCHEFFLER S, KALUS U, ROJEWSKI, $M$ et al. Comparative Analysis of Different Platelet Lysates and Platelet Rich Preparations to Stimulate Tendon Cell Biology: An In Vitro Study. Int. J. Mol. Sci.19:212. 2018.

SIERRA ALP, ARANEGUI RO, ARES MM, MARTÍNEZ JLQ, GONZÁLEZ JMM. Quantification of growth factors by using a new system for obtaining plateletrich plasma. Med Oral Patol Oral Cir Bucal. I6(4): 614-8, 20II.

STEVENS J, KHETARPAL S. Platelet-rich plasma for androgenetic alopecia: A review of the literature and proposed treatment protocol. International Journal of Women's Dermatology. 5, 46-51.2019.

TAHRIRIAN MA, MOEZI M, MOTIFIFARD M, NEMATI M, NEMATI A. Ultrasound guided platelet-rich plasma injection for the treatment of rotator cuff tendinopathy. Adv Biomed Res. 5:200.2016.

VASCONCELOS RCF, AZUAGA K, ARENAS GCF, VASCONCELOS JGF, BORELLI NS. A aplicação do plasma rico em plaquetas no tratamento da alopecia androgenetica. Surg Cosmet Dermatol. 8(2):130-7. 2015.

VASCONCELOS RCF, AZUAGA K, EID RT, ARENAS GCF. A utilização de plasma rico em plaquetas no tratamento da alopecia areata de difícil controle. Surg Cosmet Dermatol. 8 (4 Supl. I):S56-9. 2016.

YIN WJ, XU HT, SHENG JG, AN ZQ, GUO SC, XIE XT, ZHANG CQ. Advantages of Pure Platelet-Rich Plasma Compared with Leukocyte- and PlateletRich Plasma in Treating Rabbit Knee Osteoarthritis. Med Sci Monit. 22:1280-1290. 2016. 